\title{
Intranasal dexmedetomidine is an effective sedative agent for electroencephalography in children
}

Hang Chen, Fei Yang, Mao Ye, Hui Liu, Jing Zhang, Qin Tian, Ruiqi Liu, Qing Yu, Shangyingying Li and Shengfen Tu*

\begin{abstract}
Background: Intranasal dexmedetomidine (DEX), as a novel sedation method, has been used in many clinical examinations of infants and children. However, the safety and efficacy of this method for electroencephalography (EEG) in children is limited. In this study, we performed a large-scale clinical case analysis of patients who received this sedation method. The purpose of this study was to evaluate the safety and efficacy of intranasal DEX for sedation in children during EEG.
\end{abstract}

Methods: This was a retrospective study. The inclusion criteria were children who underwent EEG from October 2016 to October 2018 at the Children's Hospital affiliated with Chongqing Medical University. All the children received $2.5 \mathrm{\mu g} \cdot \mathrm{kg}^{-1}$ of intranasal DEX for sedation during the procedure. We used the Modified Observer Assessment of Alertness/Sedation Scale (MOAA/S) and the Modified Aldrete score (MAS) to evaluate the effects of the treatment on sedation and resuscitation. The sex, age, weight, American Society of Anesthesiologists physical status (ASAPS), vital signs, sedation onset and recovery times, sedation success rate, and adverse patient events were recorded.

Results: A total of 3475 cases were collected and analysed in this study. The success rate of the initial dose was $87.0 \%$ (3024/3475 cases), and the success rate of intranasal sedation rescue was 60.8\% (274/451 cases). The median sedation onset time was 19 mins (IQR: 17-22 min), and the sedation recovery time was 41 mins (IQR: 36-47 min). The total incidence of adverse events was $0.95 \%$ (33/3475 cases), and no serious adverse events occurred.

Conclusions: Intranasal DEX $\left(2.5 \mu \mathrm{g} \cdot \mathrm{kg}^{-1}\right)$ can be safely and effectively used for EEG sedation in children.

Keywords: Children, Electroencephalography, Intranasal dexmedetomidine, Sedation

\section{Background}

Electroencephalography (EEG) is an important tool for the clinical diagnosis of epilepsy, mental disorders, intracranial tumours and other nervous system diseases. However, for children who have difficulty falling asleep due to a lack of cooperation or anxiety before the examination, a satisfactory form of

\footnotetext{
* Correspondence: 595494227@qq.com

Department of Anesthesiology, Children's Hospital of Chongqing Medical University, No.136 Zhongshan 2nd Road, Yuzhong District, Chongqing, People's Republic of China
}

sedation can make the examination process more efficient and comfortable [1].

Many sedative drugs have been used for paediatric sedation in the past, but the use of many sedative drugs for EEG is controversial. For example, ketamine, propofol and sevoflurane can affect brain waves, which may lead to an incorrect diagnosis based on an EEG. Midazolam and chloral hydrate have been used in the past but have some shortcomings with regard to safety and sedative efficacy, respectively [2].

C C The Author(s). 2020 Open Access This article is licensed under a Creative Commons Attribution 4.0 International License, which permits use, sharing, adaptation, distribution and reproduction in any medium or format, as long as you give appropriate credit to the original author(s) and the source, provide a link to the Creative Commons licence, and indicate if changes were made. The images or other third party material in this article are included in the article's Creative Commons licence, unless indicated otherwise in a credit line to the material. If material is not included in the article's Creative Commons licence and your intended use is not permitted by statutory regulation or exceeds the permitted use, you will need to obtain permission directly from the copyright holder. To view a copy of this licence, visit http://creativecommons.org/licenses/by/4.0/ The Creative Commons Public Domain Dedication waiver (http://creativecommons.org/publicdomain/zero/1.0/) applies to the data made available in this article, unless otherwise stated in a credit line to the data. 
Dexmedetomidine (DEX) is a highly selective alpha2 adrenergic receptor agonist that mainly acts on the alpha-2 receptor in the spinal cord and the nucleus of the locus coeruleus. DEX has little influence on haemodynamics or respiratory inhibition and has a short half-life [3, 4]. Previous studies have shown that DEX interferes little with the basic background waves of the brain; it slightly increases theta, alpha and beta activities but has no effect on the detection of an epileptic discharge $[5,6]$. In addition, DEX produces a state similar to natural sleep, which can be reversed with conversation, enabling clinicians to assess a child's cognitive status after the completion of an EEG examination [7]. As animal studies have shown, drugs can be administered through the nasal cavity, which can effectively reduce first-pass elimination, and the drug can more efficiently enter the brain through the nervous olfactory system [8]. The overall bioavailability of DEX in children with intranasal administration was reported to be $84 \%$ [9]. The use of DEX alone in paediatric sedation provides adequate sedation $[10,11]$. Thus, DEX is a sedative suitable for EEG, no comprehensive studies have been performed regarding the safety and effective dose of DEX. Evaluating the safety and efficacy of $2.5 \mu \mathrm{g} \cdot \mathrm{kg}^{-1}$ intranasal DEX was the main objective of this study.

An increasing number of studies have reported the use of DEX in clinical practice. In children under deep sedation, failure to strictly meet the fasting requirement before anaesthesia did not lead to an increase in adverse events [12]. In contrast, prolonged fasting may cause anxiety in children, making them difficult to placate and leading to a reduction in sedation success rate [13].

The purpose of this study was to evaluate the safety and efficacy of intranasal DEX in paediatric EEG sedation and to provide a reference for clinical sedative use in paediatrics.

\section{Methods}

\section{Patient population}

This was a retrospective research study that was approved by the Ethics Committee of the Children's Hospital affiliated with Chongqing Medical University. Our study retrospectively analysed children who underwent EEG from October 2016 to October 2018 at our hospital. Patients were sedated with $2.5 \mu \mathrm{g} \cdot \mathrm{kg}^{-1}$ intranasal DEX.

\section{Sedation method}

The inclusion criteria for this study were children who underwent EEG in our hospital who received $2.5 \mu \mathrm{g} \cdot \mathrm{kg}^{-1}$ of intranasal DEX. Children were excluded when they met any of the following criteria: (1) A history of allergy to DEX, (2) difficult airway, (3) anatomical structural deformity of the nasal cavity, (4) severe liver or renal insufficiency and (5) severe bradycardia or atrioventricular block above II degree type 2 .

Our standard sedation procedure was as follows. Children needed to fast for at least $1 \mathrm{~h}$ before sedation. An anaesthesiologist evaluated the patient's general condition, history of the present illness, previous medical history, surgical history, allergy history and sedative history. Then, the anaesthesiologist created an appropriate sedative plan, and an informed consent form was signed. The child was placed in a supine position and attended by a guardian, and a nurse administered a nasal drip of $2.5 \mu \mathrm{g} \cdot \mathrm{kg}^{-1}$ DEX to the child. All the children remained lying flat for 1-2 min after the medicine was administered while we gently massaged the alae of the nose of the children to facilitate DEX absorption by the nasal mucosa. We used the Modified Observer Assessment of Alertness/Sedation Scale (MOAA/S) [14] (Table 1) to evaluate the children's sedation state. Successful sedation was defined as an MOAA/S score less than or equal to 3 within 30 mins after the first dose of DEX. If the MOAA/S score was greater than 3 within 30 min after the first dose of DEX, an additional $1 \mu \mathrm{g} \cdot \mathrm{kg}^{-1}$ intranasal DEX was given as a "rescue" dose. If the EEG could still not be completed, inhaled sevoflurane were administered to allow the examination to be completed, which we defined as failed sedation. After drug administration, the anaesthesiologist not only assessed the child's sedation level but also recorded heart rate (HR), pulse oxygen saturation $\left(\mathrm{SpO}_{2}\right)$, and the occurrence of adverse events, which referred to postoperative nausea and vomiting (PONV), bradycardia, $\mathrm{SpO}_{2}$ reduction, etc. The EEG was performed after successful sedation, while the attending physician used a portable monitor to track the patient's $\mathrm{HR}$ and $\mathrm{SpO}_{2}$. We defined the onset time of sedation as the time from drug administration to successful sedation. Recovery time was defined as the time from successful sedation to recovery. After the examination, the children were sent back to the sedation recovery room for further observation. Patients were discharged upon attaining a Modified Aldrete score (MAS) [15] (Table 2)

Table 1 Modified Observer's Assessment of Alertness/Sedation

Scale

\begin{tabular}{ll}
\hline Response & Score \\
\hline Agitated & 6 \\
Responds readily to name spoken in normal tone & 5 \\
Lethargic response to name spoken in normal tone & 4 \\
Responds only after name is called loudly and repeatedly & 3 \\
Responds only after mild prodding or shaking & 2 \\
Does not respond to mild prodding or shaking & 1 \\
Does not respond to a deep stimulus & 0 \\
\hline
\end{tabular}


Table 2 Modified Aldrete score

\begin{tabular}{ll}
\hline Criterion & Score \\
\hline Activity & 2 \\
Move 4 extremities voluntarily or on command & 1 \\
Move 2 extremities voluntarily or on command & 0 \\
Unable to move extremities voluntarily or on command & \\
Respiration & 2 \\
Able to breathe deeply, cough, and/or cry & 1 \\
Dyspnoea or limited breathing & 0 \\
Apnoeic & \\
Circulation & 2 \\
Blood pressure \pm 20 mmHg of preanaesthetic value & 1 \\
Blood pressure \pm 21 to 49 mmHg of preanaesthetic value & 0 \\
Blood pressure \pm 50 mmHg of preanaesthetic value & \\
Consciousness & 2 \\
Fully awake & 1 \\
Arousable on calling & \\
Unresponsive & \\
Oxygen saturation & \\
Able to maintain oxygen saturation > 92\% on room air & 2 \\
Needs oxygen inhalation to maintain oxygen saturation > 90\% & 1 \\
Oxygen saturation < $90 \%$ even with oxygen supplementation & 0 \\
\hline
\end{tabular}

of 9 or upon reaching the following states: (1) stable cardiovascular function and unobstructed respiratory tract; (2) awakened easily, with protective airway reflexes intact; (3) ability to communicate with others (age-appropriate assessment); (4) able to sit up unassisted (ageappropriate assessment); (5) for very small children or children with disabilities who were unable to exhibit the usual expected responses, a return to pre-sedation response levels or to as close to normal as possible; and (6) adequate hydration status.

\section{Data collection}

Sex, age, weight, American Society of Anesthesiologists physical status (ASAPS), vital signs, sedation onset and recovery times, success of sedation, adverse events, etc. were collected and recorded in Microsoft Excel 2010.

\section{Adverse events and handling}

Adverse events were classified as severe or minor, and the occurrences of adverse events was recorded. The serious adverse events were: (1) emergency airway intervention (the use of tracheal intubation or the placement of airway aids, such as oropharynx or larynx masks); (2) laryngospasm; (3) reflux aspiration; (4) severe arrhythmia; (5) respiratory and cardiac arrest. The minor adverse reaction events were as follows: (1) bradycardia, defined as a heart rate deceleration of greater than $20 \%$ of the normal age- adjusted rate during sedation and need drug intervention (treated with atropine intravenously); (2) a significant oxygen saturation decrease, defined as an $\mathrm{SpO}_{2}$ of less than 90\%; (3) upper respiratory tract obstruction (open airway; can be reversed with mask oxygen); (4) PONV (tilt the child's head to one side while removing vomit from the mouth); (5) recovery delay, defined as a sedation recovery time $>2 \mathrm{~h}$; and (6) rash.

\section{Statistical analysis}

Quantitative data with a normal distribution are described with the mean \pm standard deviation or median and interquartile ranges. Categorical variables are represented by a number, and the rate and 95\% confidence interval $(\mathrm{CI})$ were calculated. All clinical data were analysed using SPSS 17.0 for Windows (SPSS Inc., Chicago, IL, USA).

\section{Results}

\section{Demographics and sedation characteristics}

This study included 3475 cases of children who were examined by EEG from October 2016 to October 2018. There were 2229 (64.1\%) males and 1246 (35.9\%) females. The age of the children was $61.7 \pm 38.9$ months. The weight of the children was $19.5 \pm 11.4 \mathrm{~kg}$. In total, 1914 patients $(55.1 \%)$ were assigned to ASAPS Class 1, 1523 patients $(43.8 \%)$ were assigned to ASAPS Class 2 and 38 patients (1.1\%) were assigned to ASAPS Class 3, as shown in Table 3.

\section{Success rate of sedation}

The success rate of the initial DEX dose was $87.0 \%$ (3024/3475 cases), and the success rate of intranasal sedation rescue was $60.8 \%$ (274/451 cases).

\section{The time of sedation and examination}

The median sedation onset time was 19 mins (IQR: 17$22 \mathrm{~min}$ ), and the sedation recovery time was 41 mins (IQR: 36-47 $\mathrm{min}$ ).

Table 3 Demographics and sedation characteristics

\begin{tabular}{ll}
\hline Characteristics & Value \\
\hline $\mathrm{N}$ & 3475 \\
Sex (M/F) & $2229(64.1) / 1246(35.9)$ \\
Age (months) & $61.7 \pm 38.9$ \\
Weight (kg) & $19.5 \pm 11.4$ \\
ASAPS & \\
1 & $1914(55.1)$ \\
2 & $1523(43.8)$ \\
3 & $38(1.1)$ \\
4 & $0(0)$ \\
\hline
\end{tabular}

Age and weight are expressed as the mean \pm standard deviation; the other data are expressed as numbers (\%) 


\section{Adverse events}

The total rate of adverse events in this study was $0.95 \%$ (33/3475 cases). Among the adverse events, no serious adverse events occurred. Among the minor adverse events, PONV was found in 20 cases $(0.58,95 \%$ CI: 0.3$0.8 \%) ; \mathrm{SpO}_{2}$ was reduced in 6 cases $(0.17,95 \% \mathrm{CI}$ : 0 $0.3 \%)$; upper respiratory tract obstruction was observed in 3 cases $(0.09,95 \%$ CI: $0-0.2 \%)$; rash was observed in 2 cases $(0.06,95 \%$ CI: $0-0.1 \%)$; heart rate decreased by more than $20 \%$ of the normal age-adjustment and drug intervention was required in 1 case $(0.03,95 \%$ CI: $0-$ $0.1 \%)$; and recovery delay occurred in 1 case $(0.03,95 \%$ CI: $0-0.1 \%)$, as shown in Table 4.

\section{Discussion}

In the past, many sedative drugs were used clinically for moderate and deep sedation during paediatric examinations, but EEG examination is unique. Many sedative drugs that act on the central nervous system interfere with brain waves. Some studies have shown that ketamine can selectively inhibit the thalamic neocortex system and activate the medulla oblongata and limbic system as well as indirectly excite the brain waves and increase the theta wave due to the "separation anaesthesia" effect of ketamine [16]. A small dose of propofol can increase beta waves, and a high dose of propofol increases the delta wave frequency due to the double action of propofol at different dosages [17]. Sevoflurane affects the number of slow delta and alpha waves [18]. The sedative drugs midazolam and chloral hydrate were used in the past; while they exhibited little interference with EEG, they had a long half-life, many side effects, high sedation failure rates and other undesirable characteristics $[19,20]$. DEX has a good sedative effect when it is administered via intranasal administration or intravenous injection [21]. Additionally, DEX has been widely used for sedation before paediatric examinations [22]. Because DEX is a new sedative, its use in EEG procedures is limited. We summarized the experience of intranasal DEX in EEG, which can provide a reference for clinical practice.

In our study, the success rate of the initial dose of $2.5 \mu \mathrm{g} \cdot \mathrm{kg}^{-1}$ DEX was $87.0 \%$ (3024/3475 cases). A recent study have found that $90 \%$ of the effective dose of

Table 4 Adverse events

\begin{tabular}{lll}
\hline Adverse events & $\mathrm{N}(\%)$ & $95 \% \mathrm{Cl}$ \\
\hline PONV & $20(0.58)$ & $0.30-0.80$ \\
Significant $\mathrm{SpO}_{2}$ reduction & $6(0.17)$ & $0-0.30$ \\
Upper airway obstruction & $3(0.09)$ & $0-0.20$ \\
Rash & $2(0.06)$ & $0-0.10$ \\
Bradycardia requiring drug intervention & $1(0.03)$ & $0-0.10$ \\
Delayed awakening & $1(0.03)$ & $0-0.10$ \\
\hline
\end{tabular}

intranasal DEX sedation was $3.28 \mu \mathrm{g} \cdot \mathrm{kg}^{-1}$ in children [23]. Another study found that the $50 \%$ effective dose and the 95\% effective dose of intranasal DEX increased with increasing age in patients under 3 years of age [24]. The success rate of sedation in our study was slightly lower than that in previous studies [11]. We believe the reason for this observation is that the age $(61.7 \pm 38.9$ months) of the children in this study was higher than that in previous studies. Therefore, we speculate that when older children are sedated, the initial dose can be appropriately increased to improve the sedation success rate, but further research is needed to verify the safety and efficacy of this approach. Notably, Jenny Bua found DEX is an attractive and reliable sedative in preterm neonates undergoing MRI. We also hope to further study the safety of DEX in preterm neonates during EEG [25].

In this study, no serious adverse events occurred in 3475 paediatric cases. In contrast to previous studies, there were no respiratory-related severe adverse events (such as laryngospasm and bronchospasm) while using intranasal DEX [26]. This result again confirms the safety of this sedation method.

The most common adverse event was PONV (0.58\%), which resolves on its own after rest. Through the monitoring of vital signs after sedation, we found that DEX can slow the heart rate of children [27]. Therefore, we speculate that because of the specific physiology of children, the heart rate slowed down, and the symptoms of nausea and vomiting appeared. The incidence of PONV was higher in our study than in previous studies on the use of intranasal DEX for various paediatric examinations [26]. This observation may be due to the various nervous system diseases in patients who underwent EEG in our study.

There are still some limitations to our research. First, the subjects in this study were children aged from half a month to 204 months $(61.7 \pm 38.9$ months), and there are differences in the physiological characteristics among different age groups. In addition, the anaesthetist evaluated the sedation depth of the children with external stimulation after nasal sedation, but this monitoring was not continuous, so there was a certain error while recording the onset time and recovery time of sedation; the recorded time was often longer than the actual time. Additionally, this was a retrospective study. Continuous blood pressure monitoring was not performed routinely as standard practice in hospital clinics, so we cannot report whether the children had hyper or hypotension as a possible side effect during the whole examination process, which also needs to be confirmed by prospective studies.

\section{Conclusion}

An intranasal DEX dose of $2.5 \mu \mathrm{g} \cdot \mathrm{kg}^{-1}$ for paediatric EEG examinations has a high sedation success rate, quick recovery and low incidence of adverse reactions. 


\section{Abbreviations}

ASAPS: American Society of Anesthesiologists physical status;

DEX: Dexmedetomidine; EEG: Electroencephalography; HR: Heart rate; IQR: Interquartile ranges; kg: Kilogram; MAS: Modified Aldrete Score; mg: Milligram; min: Minute; ml: Millilitre; MOAA/S: Modified Observer's Assessment of Alertness and Sedation; PONV: Postoperative nausea and vomiting; $\mathrm{SpO}_{2}$ : Pulse oxygen saturation; $\mu \mathrm{g}$ : Microgram

\section{Acknowledgements}

Not applicable.

\section{Authors' contributions}

$\mathrm{CH}$ helped design and perform the study, and this author contributed significantly to the analysis and manuscript preparation. TSF participated in the design and draft the manuscript. YF performed the quality assessment, and helped to draft the manuscript. YM performed the quality assessment. $\mathrm{LH}$ and ZJ helped to perform statistical analyses and search strategy. TQ, $L R Q, Y Q, L S Y Y$ helped to perform the study. All authors have read and approved the manuscript.

\section{Funding}

There was no funding source in this study.

\section{Availability of data and materials}

All data generated or analyzed during this study are included in this published article. Raw data are available upon reasonable request from the corresponding author.

\section{Ethics approval and consent to participate}

Ethics approval for this study (File NO. 2016124) was provided by the Institutional Review Board of Children's Hospital Affiliated with Chongqing Medical University, Chongqing, China (Chairperson Professor Lu Zhongyi) on 04 December 2016. Each child's parents signed the informed consent form.

\section{Consent for publication}

Not applicable.

\section{Competing interests}

The authors declare that they have no competing interests

\section{Received: 6 November 2019 Accepted: 3 March 2020} Published online: 07 March 2020

\section{References}

1. Keidan I, Ben-Menachem E, Tzadok M, Ben-Zeev B, Berkenstadt H. Electroencephalography for children with autistic spectrum disorder: a sedation protocol. Paediatr Anaesth. 2015;25(2):200-5.

2. Zhang H, Fang B, Zhou W. The efficacy of dexmedetomidine-remifentanil versus dexmedetomidine-propofol in children undergoing flexible bronchoscopy: a retrospective trial. Medicine. 2017;96(1):e5815.

3. Berkenbosch JW. Options and considerations for procedural sedation in pediatric imaging. Paediatric drugs. 2015;17(5):385-99.

4. Tong Y, Ren H, Ding X, Jin S, Chen Z, Li Q. Analgesic effect and adverse events of dexmedetomidine as additive for pediatric caudal anesthesia: a meta-analysis. Paediatr Anaesth. 2014;24(12):1224-30

5. Mason KP, O'Mahony E, Zurakowski D, Libenson MH. Effects of dexmedetomidine sedation on the EEG in children. Paediatr Anaesth. 2009; 19(12):1175-83.

6. Ray T, Tobias JD. Dexmedetomidine for sedation during electroencephalographic analysis in children with autism, pervasive developmental disorders, and seizure disorders. J Clin Anesth. 2008;20(5):364-8.

7. Mahmoud M, Mason KP. Dexmedetomidine: review, update, and future considerations of paediatric perioperative and periprocedural applications and limitations. Br J Anaesth. 2015;115(2):171-82.

8. Charlton ST, Whetstone J, Fayinka ST, Read KD, Illum L, Davis SS. Evaluation of direct transport pathways of glycine receptor antagonists and an angiotensin antagonist from the nasal cavity to the central nervous system in the rat model. Pharm Res. 2008;25(7):1531-43.

9. Miller JW, Balyan R, Dong M, Mahmoud M, Lam JE, Pratap JN, Paquin JR, Li BL, Spaeth JP, Vinks A, et al. Does intranasal dexmedetomidine provide adequate plasma concentrations for sedation in children: a pharmacokinetic study. Br J Anaesth. 2018;120(5):1056-65.

10. Yuen VM, Hui TW, Irwin MG, Yao TJ, Wong GL, Yuen MK. Optimal timing for the administration of intranasal dexmedetomidine for premedication in children. Anaesthesia. 2010;65(9):922-9.

11. Baier NM, Mendez SS, Kimm D, Velazquez AE, Schroeder AR. Intranasal dexmedetomidine: an effective sedative agent for electroencephalogram and auditory brain response testing. Paediatr Anaesth. 2016;26(3):280-5.

12. Clark M, Birisci E, Anderson JE, Anliker CM, Bryant MA, Downs C, Dalabih A. The risk of shorter fasting time for pediatric deep sedation. Anesth Essays Res. 2016;10(3):607-12.

13. Keidan I, Gozal D, Minuskin T, Weinberg M, Barkaly H, Augarten A. The effect of fasting practice on sedation with chloral hydrate. Pediatr Emerg Care. 2004;20(12):805-7.

14. Shetty SK, Aggarwal G. Efficacy of intranasal Dexmedetomidine for conscious sedation in patients undergoing surgical removal of impacted third molar: a double-blind Split mouth study. J Maxillofac Oral Surg. 2016; 15(4):512-6.

15. Mason KP, Robinson F, Fontaine P, Prescilla R. Dexmedetomidine offers an option for safe and effective sedation for nuclear medicine imaging in children. Radiology. 2013;267(3):911-7.

16. Ahnaou A, Huysmans H, Biermans R, Manyakov NV, Drinkenburg W. Ketamine: differential neurophysiological dynamics in functional networks in the rat brain. Transl Psychiatry. 2017;7(9):e1237.

17. Ke JD, Xu M, Wang PP, Wang M, Tian M, Chen ACN. Influence of propofol on the electroencephalogram default mode network in patients of advanced age. J Int Med Res. 2018;46(11):4660-8.

18. de Heer IJ, Bouman SJM, Weber F. Electroencephalographic (EEG) density spectral array monitoring in children during sevoflurane anaesthesia: a prospective observational study. Anaesthesia. 2019;74(1):45-50.

19. Sheta SA, Al-Sarheed MA, Abdelhalim AA. Intranasal dexmedetomidine vs midazolam for premedication in children undergoing complete dental rehabilitation: a double-blinded randomized controlled trial. Paediatr Anaesth. 2014;24(2):181-9.

20. Yuen VM, Li BL, Cheuk DK, Leung MKM, Hui TWC, Wong IC, Lam WW, Choi SW, Irwin MG. A randomised controlled trial of oral chloral hydrate vs. intranasal dexmedetomidine before computerised tomography in children. Anaesthesia. 2017;72(10):1191-5.

21. Mason KP, Lubisch NB, Robinson F, Roskos R. Intramuscular dexmedetomidine sedation for pediatric MRI and CT. AJR Am J Roentgenol. 2011;197(3):720-5.

22. Sulton C, McCracken C, Simon HK, Hebbar K, Reynolds J, Cravero J, Mallory M, Kamat P. Pediatric procedural sedation using Dexmedetomidine: a report from the pediatric sedation research consortium. Hospital pediatrics. 2016; 6(9):536-44

23. Liu H, Sun M, Zhang J, Tian Q, Yu Q, Liu Y, Yang F, Li S, Tu S. Determination of the $90 \%$ effective dose of intranasal dexmedetomidine for sedation during electroencephalography in children. Acta Anaesthesiol Scand. 2019; 63(7):847-52.

24. Yu Q, Liu Y, Sun M, Zhang J, Zhao Y, Liu F, Li S, Tu S. Median effective dose of intranasal dexmedetomidine sedation for transthoracic echocardiography in pediatric patients with noncyanotic congenital heart disease: an up-anddown sequential allocation trial. Paediatr Anaesth. 2017;27(11):1108-14.

25. Bua J, Massaro M, Cossovel F, Monasta L, Brovedani P, Cozzi G, Barbi E, Demarini S, Travan L. Intranasal dexmedetomidine, as midazolam-sparing drug, for MRI in preterm neonates. Paediatr Anaesth. 2018;28(8):747-8.

26. Yang F, Liu Y, Yu Q, Li S, Zhang J, Sun M, Liu L, Lei Y, Tian Q, Liu H, et al. Analysis of 17948 pediatric patients undergoing procedural sedation with a combination of intranasal dexmedetomidine and ketamine. Paediatr Anaesth. 2019;29(1):85-91.

27. Chrysostomou C, Komarlu R, Lichtenstein S, Shiderly D, Arora G, Orr R, Wearden PD, Morell VO, Munoz R, Jooste EH. Electrocardiographic effects of dexmedetomidine in patients with congenital heart disease. Intensive Care Med. 2010;36(5):836-42.

\section{Publisher's Note}

Springer Nature remains neutral with regard to jurisdictional claims in published maps and institutional affiliations. 